\title{
SUPERSYMMETRY, HOMOLOGY WITH TWISTED COEFFICIENTS AND $n$-DIMENSIONAL KNOTS
}

\author{
EIJI OGASA
}

\begin{abstract}
Let $n$ be any natural number. Let $K$ be any $n$-dimensional knot in $S^{n+2}$. We define a supersymmetric quantum system for $K$ with the following properties. We firstly construct a set of functional spaces (spaces of fermionic \{resp. bosonic\} states) and a set of operators (supersymmetric infinitesimal transformations) in an explicit way. Thus we obtain a set of the Witten indexes for $K$. Our Witten indexes are topological invariants for $n$-dimensional knots. Our Witten indexes are not zero in general. If $K$ is equivalent to the trivial knot, all of our Witten indexes are zero. Our Witten indexes restrict the Alexander polynomials of $n$-knots. If one of our Witten indexes for an $n$-knot $K$ is nonzero, then one of the Alexander polynomials of $K$ is nontrivial. Our Witten indexes are connected with homology with twisted coefficients.

Roughly speaking, our Witten indexes have path integral representation by using a usual manner of supersymmetric theory.
\end{abstract}

\section{INTRODUCTION AND OUR MAIN CLAIMS}

When we discuss time-space, we often regard time-space as a manifold other than $\mathbb{R}^{4}$ especially after Einstein's general theory of relativity [7]. This kind of discussion is essentially useful both for physics and for mathematics.

As is well-known, the papers [2] [25] state that: if we discuss the Witten index for a kind of supersymmetric quantum theory on a manifold, then we get the Atiyah-Singer index of an operator on some bundles on the manifold, which gives a topological invariant of the manifold in some cases.

Let $n$ be any natural number. Let $K$ be any $n$-dimensional knot in $S^{n+2}$ (see e.g. [4] [13] [14] 22] for $n$-knots). In this paper we consider a question whether we can make a model of supersymmetric quantum theory associated with the $n$-knot $K$ with the following properties(1)-(4):

(1) Our Witten indexes for our supersymmetric infinitesimal operators are topological invariants of $n$-knots $K$.

(2) All of our Witten indexes are not zero in general.

Keywords: supersymmetry, supersymmetry breaking, the Witten index, the euler number, homology with twisted coefficients, deRham cohomology, $n$-dimensional knots, cyclic covering spaces, the Alexander polynomials for $n$-knots, space of states, supersymmetric infinitesimal transformation . 
(3) All of our Witten indexes are zero for the trivial $n$-knot.

(4) The space of fermionic (resp. bosonic) states in this model is infinite dimensional vector space.

In $§ 3$ we give a positive answer. We construct such a model in an explicit way. We construct a set of functional spaces (spaces of fermionic \{resp. bosonic $\}$ states), a set of operators (supersymmetric infinitesimal transformations, and Hamiltonians). Our Witten indexes have the above properties (1)-(4) and, furthermore, have the following properties(5)(6):

(5) Our Witten indexes restrict the Alexander polynomial for $n$-dimensional knots.

(6) Our Witten indexes restrict the homology of $S^{n+2}-K$ with a twisted coefficient.

The Witten indexes have path integral representation (see [2]). Roughly speaking, hence our way gives path integral representation to some topological invariants for $n$-knots.

The study of $n$-dimensional knots and $n$-dimensional links has a long history and many fruitful results (see e.g. [4] [13, [14 [22]). Note that it is known that, for any natural number $n$, there are countably infinitely many nonequivalent $n$-knots.

\section{Review of the Witten Index}

In order to define our Witten indexes, we review the Witten index (see [2] 25]). But we do not mind how rigorous our explanation is from mathematical viewpoints.

Let $\mathcal{H}$ be an infinite dimensional vector space (over $\mathbb{C}$ ) which is a subset of a set of sections of a $\mathbb{C}^{n}$-vector bundle on a manifold. Suppose $\mathcal{H}$ has a metric. Let $H$ be an operator to act on $\mathcal{H}$. Suppose that this space $\mathcal{H}$ and this operator $H$ satisfy the following:

(1) There is a direct sum $\mathcal{H}=\mathcal{H}_{-} \oplus \mathcal{H}_{+}$.

(2) There is an operator $Q: \mathcal{H} \rightarrow \mathcal{H}$ such that $Q\left(\mathcal{H}_{+}\right) \subset \mathcal{H}_{-}$and $Q\left(\mathcal{H}_{-}\right)=0$. Let $Q^{*}$ denote the adjoint operator of $Q$. It holds that $Q^{*}\left(\mathcal{H}_{-}\right) \subset \mathcal{H}_{+}$and $Q^{*}\left(\mathcal{H}_{+}\right)=0$. Thus we have:

$$
0 \underset{Q^{*}}{\longleftarrow} \mathcal{H}_{+} \underset{Q^{*}}{\stackrel{Q}{\rightleftarrows}} \mathcal{H}_{-} \stackrel{Q}{\longrightarrow} 0
$$

where $Q$ also denotes $\left.Q\right|_{\mathcal{H}_{+}}\left(\operatorname{resp} .\left.Q\right|_{\mathcal{H}_{-}}\right)$and $Q^{*}$ also denotes $\left.Q^{*}\right|_{\mathcal{H}_{+}}\left(\right.$resp. $\left.\left.Q^{*}\right|_{\mathcal{H}_{-}}\right)$. (3) $H=Q Q^{*}+Q^{*} Q$

In literatures of Physics, $H$ is called the Hamiltonian, $\mathcal{H}$ is called a sapce of states. $\mathcal{H}_{+}$ (resp. $\mathcal{H}_{-}$) is called a space of bosonic (resp. fermionic) states under some conditions (see the second Note from the following). $Q$ is a supercharge or an infinitesimal transformation of a supersymmetric transformation. Note that then the supersymmetric transformation is $e^{i \epsilon Q}$, where $\epsilon$ has some properties. @ See [2] 24] etc for detail. 
Let $F$ be an operator to act on $\mathcal{H}$ such that we have $F|q>=| q>$ and $(-1)^{F}|q>=(-1)| q>$ for any vector $\mid q>\in \mathcal{H}_{-}$and that $F \mid p>=0$ and $(-1)^{F}|p>=| p>$ for any vector $\mid p>\in \mathcal{H}_{+}$, where note that $(-1)^{F}=e^{i \pi F}$. We define the Witten index for a set of $\mathcal{H}, \mathcal{H}_{-}, \mathcal{H}_{+}, H, Q$ to be $\operatorname{dim}\left(\left.\operatorname{Ker} Q\right|_{\mathcal{H}_{+}}\right)_{-}$ $\operatorname{dim}\left(\left.\operatorname{Ker} Q^{*}\right|_{\mathcal{H}_{-}}\right)$. The Witten index is often denoted by $\operatorname{tr}(-1)^{F}$ or $\operatorname{tr}\left\{(-1)^{F} e^{-\beta H}\right\}$.

Note. This operation 'tr' depends not only $\mathcal{H}_{+}, \mathcal{H}_{-}, F$ but also on $Q$. This ' $\operatorname{tr}$ ' is essentially same as 'tr' in partition functions (see e.g. (4.5) (5.150) of [23]).

Let $n_{b}=\operatorname{dim}\left(\left.\operatorname{Ker} Q\right|_{\mathcal{H}_{+}}\right)$and $n_{f}=\operatorname{dim}\left(\left.\operatorname{Ker} Q^{*}\right|_{\mathcal{H}_{-}}\right)$. Then the Witten index is $n_{b}-n_{f}$. It is known that: Supersymmetry is broken if and only if $n_{b}=n_{f}=0$. If supersymmetry is broken, then the Witten index is zero. That is, if the Witten index is nonzero, supersymmetry is not broken.

Note. In order to make $\mathcal{H}_{+}$and $\mathcal{H}_{-}$more physics-like, we impose that the elements of $\mathcal{H}_{-}$(resp. $\mathcal{H}_{+}$) satisfy (resp. do not satisfy ) the Pauli exclusion principle. (Some researchers only call the elements of $\mathcal{H}_{-}$(resp. $\mathcal{H}_{+}$) fermions (resp. bosons) only under this condition. ) In the case in 2] 25] where the Witten index is the euler number, the product of differential forms gives such properties. However, in the case in 22 25] where the Witten index is the signature, the product of differential forms does not give such properties.

The papers [2] 25] say that the Witten index is equal to the Atiyah-Singer index of the operator $Q: \mathcal{H}_{+} \rightarrow \mathcal{H}_{-}$under some conditions. Recall that the Atiyah-Singer index is $\operatorname{dim}\left(\left.\operatorname{Ker} Q\right|_{\mathcal{H}_{+}}\right)-\operatorname{dim}\left(\left.\operatorname{Ker} Q^{*}\right|_{\mathcal{H}_{-}}\right)$. See 3$]$.

Note. 3] says that the Atiyah-Singer index theorem holds for elliptic operators of any order. [2] explanes that, for some kinds of oprators of the first order, the Atiyah-Singer index theorem is represented by a path integral. (The case of the first order elliptic operator is the most important case in today's physics.). 2])

The Witten index $\operatorname{tr}\left\{(-1)^{F} e^{-\beta H}\right\}$ has path integral representation (see (2.5) (3.2) of

$$
\operatorname{tr}\left\{(-1)^{F} e^{-\beta H}\right\}=\int_{\mathrm{PBC}} \mathcal{D} \phi \mathcal{D} \psi \exp \left[\int_{o}^{\beta} d t L\right] .
$$

See e.g. $\$ 5.5$ of [23] and [1] for APBC (anti-periodic boundary condition) and PBC. (periodic boundary condition). If $\mathcal{H}_{-}\left(\right.$resp. $\left.\mathcal{H}_{+}\right)$does not have an anti-commutative (a commutative) product, the boundary condition is diffrent form the above. See e.g. (4.5) of $[2]$.

Partition functions $\operatorname{tr}\left\{e^{-\beta H}\right\}$ are represented by path integrals. See (4.5) (4.12) (5.150) (5.151) (5.152) in [23. In the case of $\operatorname{tr}\left\{(-1)^{F} e^{-\beta H}\right\},(-1)^{F}$ makes the condition PBC. If we do not have $(-1)^{F}$, we have the condition APBC.

The domain of this path integral is 
$\mathcal{S}=\{f \mid f:[0, t] \rightarrow E, f(0)=f(t)$ or $f(0)=-f(t)$ holds by the boundary condition. $\}$, where $t \in \mathbb{R}$, and $[0, t]=\{s \mid 0 \leqq s \leqq t, t \in \mathbb{R}\}, E$ is the total space of a fiber bundle over a manifold $M$ with fiber $\mathbb{G}^{n}$. Here, $\mathbb{G}$ is the one dimensional vector space over complex Grassmann number and $\mathbb{G}^{n}$ means $n$-dimensional vector space over complex Grassmann number. See $\S 5$ of [23] for path integral representaions for fermions by using Grassmann variables, and ones for supersymmetry.

The notation $\int \mathcal{D} x \mathcal{D} y$ is derived from the fact that, in a chart $U \times \mathbb{G}^{n}$ of the $\mathbb{G}^{n}$ bundle, an element in $\mathcal{S}$ is represented by $[0,1] \ni t \rightarrow(\phi, \psi) \in U \times \mathbb{G}^{n}$.

It holds that $\mathcal{S}=\oplus_{i} A^{i}(M)$ for a $\mathbb{G}^{n}$-bundle. It holds that the Witten index is the euler number of $M$ for a combination of a Lagrangian and a $\mathbb{G}^{n}$-bundle.

\section{Our Witten indexes}

We make our Witten indexes.

Let $K$ be an $n$-knot in $S^{n+2}$. Let $M^{\mu}$ be the $\mu$-fold branched covering space of $S^{n+2}$ along $K(\mu \in \mathbb{N}-\{1\})$. See P.292 of [22] for branched covering spaces. We review it roughly in the second paragraph from the follwoing.

Let $X=S^{n+2}-\operatorname{Int} N(K)$. Let $\rho$ be the homomorphism $\pi_{1} X \rightarrow H_{1}(X ; \mathbb{Z})$ which is obtained by the abelianization. Here, note that $H_{1}(X ; \mathbb{Z}) \cong \mathbb{Z}$ and that the generator of $H_{1}(X ; \mathbb{Z}) \cong \mathbb{Z}$ is determined by the orientation of $K$ and that of $S^{n+2}$. Let $\tilde{X}^{\infty}$ be the covering space of $X$ associated with $\rho$. Let $\alpha_{\mu}: \mathbb{Z} \rightarrow \mathbb{Z}_{\mu}$ be the natural epimorphism such that $\alpha_{\mu}$ carries the generator to the generator, where we fix the generator of $\mathbb{Z}_{\mu}$. Let $\rho_{\mu}=\alpha_{\mu} \circ \rho: \pi_{1} X \rightarrow \mathbb{Z}_{\mu}$. Let $\tilde{X}^{\mu}$ be the covering space of $X$ associated with $\rho_{\mu}$.

The branched covering space $M^{\mu}$ has the following property: There is a continuous map $p: M^{\mu} \rightarrow S^{n+2}$ such that

(1) The map $\left.p\right|_{p^{-1}(K)}: p^{-1}(K) \rightarrow K$ is a homeomorphism map.

(2) It holds that $\left[M^{\mu}-p^{-1}(K)\right] \cong \operatorname{Int} \widetilde{X}^{\mu}$ and $\left[S^{n+2}-K\right] \cong \operatorname{Int} X$.

(3) The map $\left.p\right|_{\left[M^{\mu}-p^{-1}(K)\right]}:\left[M^{\mu}-p^{-1}(K)\right] \rightarrow\left[S^{n+2}-K\right]$ is same as the map $\left.\rho_{\mu}\right|_{\operatorname{Int} \widetilde{X}^{\mu}}: \operatorname{Int} \widetilde{X}^{\mu} \rightarrow \operatorname{Int} X$.

It is known that, if $\mu \neq \mu^{\prime}$, then $M^{\mu}$ is not diffeomorphic to $M^{\mu^{\prime}}$ in general. Note that the above branched covering space is a kind of generalization of branched covering spaces of Rieman surfaces in complex analysis.

We use the deRham cohomology (see 6]). Let $M$ be an oriented closed manifold with a metric. Let $*$ denote the Hodge (star) operator $A^{i}(M) \rightarrow A^{m-i}(M)$. We define an operator $d^{*}: A^{i}(M) \rightarrow A^{i-1}(M)$ so that $d^{*}=(-1)^{i}\left(*^{-1}\right) d *$. Then it holds that $d^{*} d^{*}=0$. For $\alpha, \beta \in A^{i}(M)$, we define an inner product $\left\langle\alpha, \beta>=\int_{M} \alpha \wedge * \beta\right.$. Then it holds that $<d \alpha, \beta>=<\alpha, d^{*} \beta>$. 
We define our Witten indexes for $n$-knots $K$. We define two kinds of indexes, the $(2 k, 2 k+1 ; \mu)$-Witten index $(0 \leqq 2 k \leqq 2 k+1 \leqq n+2, k \in \mathbb{Z})$ and the $(2 k, 2 k-1 ; \mu)$-Witten index $(0 \leqq 2 k-1 \leqq 2 k \leqq n+2, k \in \mathbb{Z})$.

We firstly define the $(2 k, 2 k+1 ; \mu)$-Witten index $(0 \leqq 2 k \leqq 2 k+1 \leqq n+2)$. Take the $\mu$-fold branched covering space $M^{\mu}$ of $S^{n+2}$ along $K$. Give a metric on $M^{\mu}$. Let $A^{i}$ denote $A^{i}\left(M^{\mu}\right)$.

$$
\begin{aligned}
& \left(U^{2 k}, U^{2 k+1}\right)= \\
& \begin{cases}\left(\left[\operatorname{Ker}\left(\mathrm{A}^{2 \mathrm{k}-1} \stackrel{\mathrm{d}^{*}}{\leftarrow} \mathrm{A}^{2 \mathrm{k}}\right)\right] \otimes \mathbb{C},\left[\operatorname{Ker}\left(\mathrm{A}^{2 \mathrm{k}+1} \stackrel{\mathrm{d}}{\rightarrow} \mathrm{A}^{2 \mathrm{k}+2}\right)\right] \otimes \mathbb{C}\right) & \text { if }(2 k, 2 k+1) \neq(n+1, n+2),(0,1) \\
\left(\left[\frac{A^{0}}{\operatorname{Ker}\left(\mathrm{A}^{0} \stackrel{\mathrm{d}}{\rightarrow} \mathrm{A}^{1}\right)}\right] \otimes \mathbb{C},\left[\operatorname{Ker}\left(\mathrm{A}^{1} \stackrel{\mathrm{d}}{\rightarrow} \mathrm{A}^{2}\right)\right] \otimes \mathbb{C}\right) & \text { if }(2 k, 2 k+1)=(0,1) \\
\left(\left[\operatorname{Ker}\left(\mathrm{A}^{\mathrm{n}} \stackrel{\mathrm{d}^{*}}{\leftarrow} \mathrm{A}^{\mathrm{n}+1}\right)\right] \otimes \mathbb{C},\left[\frac{\mathrm{A}^{\mathrm{n}+2}}{\operatorname{Ker}\left(\mathrm{A}^{\mathrm{n}+1} \stackrel{\mathrm{d}^{*}}{\leftarrow} \mathrm{A}^{\mathrm{n}+2}\right)}\right] \otimes \mathbb{C}\right) & \text { if }(2 k, 2 k+1)=(n+1, n+2)\end{cases}
\end{aligned}
$$

Let $\mathcal{H}_{+}=U^{2 k}$ and $\mathcal{H}_{-}=U^{2 k+1}$. We define $Q: \mathcal{H}_{+} \rightarrow \mathcal{H}_{-}$by using $d$ naturally. We define $Q^{*}$ by using $d^{*}$ naturally. We define the $(2 k, 2 k+1 ; \mu)$-Witten index to be the Witten index for these.

We next define the $(2 k, 2 k-1 ; \mu)$-Witten index $(0 \leqq 2 k-1 \leqq 2 k \leqq n+2)$.

$$
\begin{aligned}
& \left(V^{2 k}, V^{2 k-1}\right)= \\
& \begin{cases}\left.\left[\operatorname{Ker}\left(\mathrm{A}^{2 \mathrm{k}+1} \stackrel{\mathrm{d}}{\leftarrow} \mathrm{A}^{2 \mathrm{k}}\right)\right] \otimes \mathbb{C},\left[\operatorname{Ker}\left(\mathrm{A}^{2 \mathrm{k}-1} \stackrel{\mathrm{d}^{*}}{\rightarrow} \mathrm{A}^{2 \mathrm{k}-2}\right)\right] \otimes \mathbb{C}\right) & \text { if }(2 k, 2 k-1) \neq(n+2, n+1) \\
\left(\left[\frac{A^{n+2}}{\operatorname{Ker}\left(\mathrm{A}^{\mathrm{n}+2} \stackrel{\mathrm{d}^{*}}{\rightarrow} \mathrm{A}^{\mathrm{n}+1}\right)}\right] \otimes \mathbb{C},\left[\operatorname{Ker}\left(\mathrm{A}^{\mathrm{n}+1} \stackrel{\mathrm{d}^{*}}{\rightarrow} \mathrm{A}^{\mathrm{n}}\right)\right] \otimes \mathbb{C}\right) & \text { if }(2 k, 2 k-1)=(n+2, n+1)\end{cases}
\end{aligned}
$$

Let $\mathcal{H}_{+}=U^{2 k}, \mathcal{H}_{-}=U^{2 k-1}$. We define $Q: \mathcal{H}_{+} \rightarrow \mathcal{H}_{-}$by using $d^{*}$ naturally. We define $Q^{*}$ by using $d$ naturally. We define the $(2 k, 2 k-1 ; \mu)$-Witten index to be the Witten index for these.

We would explain a correspondence between our model and the real world.

(1) We give many pair of superpartners to a single $n$-knot . This situation corresponds to the fact that the real time-space is expected to have greater than one pair of superpartners. In our model, if we fix $\mu$, there are a pair of superpartners whose index is $(i, i+1)$ and another pair whose index is $(i, i-1)$. The letter $i$ appears in the two ways. This situation corresponds to the fact that: in the real timespace we expect the existence of a pair of superpartners whose index is $\left(\frac{1}{2}, 1\right)$ and another pair whose index is $\left(0, \frac{1}{2}\right)$. If the particle has the index is $\frac{1}{2}$, there are two kinds of the index of the superpartner, that is 0 or 1 . (In the real time-space the indexes represent spin of particles.)

(2) In order to make path integral representation for our Witten indexes, we restrict the space of states $A^{i}\left(M^{\mu}\right)$ and obtain a new space of states. Such restriction on 
a space of states are often done in gauge theory in QFT and string theory (See e.g. Gupta-Bleuler treatment in 99 etc.)

(3) A crucial point of the construction of our model is the fact that: a function which is an element of $\mathcal{H}_{+}$(resp. $\left.\mathcal{H}_{-}\right)$is one from $M^{\mu}$ to $\mathbb{C}$, not from $S^{n+2}$, or not from $X$. We have the following situation in complex analysis and our construction is its generalization: An operation which we represent $z \rightarrow \sqrt{z}$ should be regarded as a function from a branched covering space of a Rieman surface not as a function from the base space of the branched covering space.

From now we explain roughly that our Witten indexes satisfy the conditions in $\$ 1$ By the definition, our Witten indexes satisfy the following.

(1) Our Witten indexes are topological invariants for $n$-knots.

(2) The $(2 k, 2 k+1 ; \mu)$-Witten index is equal to

$$
\begin{cases}b_{2 k}\left(M^{\mu}\right)-b_{2 k+1}\left(M^{\mu}\right) & \text { if }(2 k, 2 k+1) \neq(n+1, n+2),(0,1) \\ b_{2 k}\left(M^{\mu}\right)-b_{2 k+1}\left(M^{\mu}\right)+1 & \text { if }(2 k, 2 k+1)=(n+1, n+2) \\ b_{0}\left(M^{\mu}\right)-b_{1}\left(M^{\mu}\right)-1 & \text { if }(2 k, 2 k+1)=(0,1)\end{cases}
$$

(3) The $(2 k, 2 k-1 ; \mu)$-Witten index is equal to

$$
\begin{cases}b_{2 k}\left(M^{\mu}\right)-b_{2 k-1}\left(M^{\mu}\right) & \text { if }(2 k, 2 k-1) \neq(n+2, n+1) \\ b_{2 k}\left(M^{\mu}\right)-b_{2 k-1}\left(M^{\mu}\right)-1 & \text { if }(2 k, 2 k-1)=(n+2, n+1)\end{cases}
$$

(4) All of our Witten indexes are zero if and only if we have $H_{l}\left(M^{\mu} ; \mathbb{R}\right)=H_{l}\left(S^{n+2} ; \mathbb{R}\right)$ for all $\mu$ and all $l$. (Because (1) and (2) hold. )

For the trivial $n$-knots, $M^{\mu}$ is diffeomorphic to the $S^{n+2}$. Hence $H_{l}\left(M^{\mu} ; \mathbb{R}\right)=H_{l}\left(S^{n+2} ; \mathbb{R}\right)$ for all $l$ and $\mu$. Hence all of our Witten indexes are zero for the trivial $n$-knots.

We discuss relations between homology with twisted coefficients and our Witten index. See e.g. Chap5 of [5] for homology with twisted coefficients for detail. Note that (co)homology with twisted coefficients is a kind of sheaf (co)homology.

Definition. Let $Y$ be a CW complex. (Note that manifolds are CW coplexes.) Let $\widetilde{Y}$ be the universal covering space. Note that the chain complex $C_{i}(\widetilde{Y})(i \in \mathbb{Z})$ is regarded as a $\mathbb{Z}\left[\pi_{1}(X)\right]$-module by using the covering transformation. Given a $\mathbb{Z}\left[\pi_{1}(X)\right]$-module $A$, form the tensor product $C_{i}(\widetilde{Y}) \otimes_{\mathbb{Z}\left[\pi_{1}(X)\right]} A$. This is a chain complex whose differential is $\partial \otimes 1$, where $\partial$ is the differential of $C_{i}(\widetilde{Y})$. The homology of this chain complex is called the homology of $Y$ with local coefficients in $A$.

Let $K$ be an $n$-knot in $S^{n+2}$. Take $X, \rho, \widetilde{X}^{\infty}, \rho_{\mu}$, and $\widetilde{X}^{\mu}$ as above. By using $\rho$ (resp. $\left.\rho_{\mu}\right)$, we can regard $\mathbb{Z}[\mathbb{Z}]\left(\right.$ resp. $\left.\mathbb{Z}\left[\mathbb{Z}_{\mu}\right]\right)$ as a $\mathbb{Z}\left[\pi_{1} X\right]$ module. Let $H_{i}(X ; \rho)\left(\operatorname{resp} . H_{i}\left(X ; \rho_{\mu}\right)\right.$ 
) be the homology with the twisted coefficient such that Shapiro's lemma (see e.g. P.100 of [5]) says :

$H_{i}(X ; \rho) \cong H_{i}\left(\widetilde{X}^{\infty} ; \mathbb{Z}\right)$, and $H_{i}(X ; \rho) \otimes \mathbb{R} \cong H_{i}\left(\widetilde{X}^{\infty} ; \mathbb{R}\right) \quad$ for $\quad i \in \mathbb{Z}$.

$H_{i}\left(X ; \rho_{\mu}\right) \cong H_{i}\left(\widetilde{X}^{\mu} ; \mathbb{Z}\right)$, and $H_{i}\left(X ; \rho_{\mu}\right) \otimes \mathbb{R} \cong H_{i}\left(\widetilde{X}^{\mu} ; \mathbb{R}\right) \quad$ for $\quad i \in \mathbb{Z} \quad \mu \in \mathbb{N}-\{1\}$.

By using the Meyer-Vietoris exact sequence, the Poincaré duality, and the universal coefficient theorem, the following three conditions are equivalent. Suppose $\mu$ is arbitrary. (1) The $(i, j ; \mu)$-Witten indexes $(i<k$ and $j<k)$ are zero@ and the $(k-1, k ; \mu)$-Witten index or the $(k, k-1 ; \mu)$-Witten index is nonzero.

(2) $H_{i}\left(M^{\mu} ; \mathbb{R}\right)=0$ for $0<i<k$ and $H_{k}\left(M^{\mu} ; \mathbb{R}\right) \neq 0$.

(3) $H_{1}\left(X ; \rho_{\mu}\right) \otimes \mathbb{R}=\mathbb{R}, H_{i}\left(X ; \rho_{\mu}\right) \otimes \mathbb{R}=0$ for $1<i<k$ and $H_{k}\left(X ; \rho_{\mu}\right) \otimes \mathbb{R} \neq 0$.

We review the definition of the Alexander polynomial for $n$-knots. See 13,22 for detail. Let $K$ be an $n$-knot in $S^{n+2}$. Make $X, \widetilde{X}^{\infty}, \widetilde{X}^{\mu}$ as above. Then we can regard $H_{i}(X ; \rho) \cong H_{i}\left(\widetilde{X}^{\infty} ; \mathbb{Z}\right)$ as $\mathbb{Z}[\mathbb{Z}]$-module by using the covering transformation associated with $\rho$. The $\mathbb{Z}[\mathbb{Z}]$-module $H_{i}(X ; \rho) \cong H_{i}\left(\widetilde{X}^{\infty} ; \mathbb{Z}\right)$ is called the $i$-Alexander module of an $n$-knot $K$ (over $\mathbb{Z}[\mathbb{Z}]$ ). Furthermore we can regard $H_{i}(X ; \rho) \otimes \mathbb{R} \cong H_{i}\left(\widetilde{X}^{\infty} ; \mathbb{R}\right)$ as $\mathbb{R}[\mathbb{Z}]$-module by using the covering transformation. The $\mathbb{R}[\mathbb{Z}]$-module $H_{i}(X ; \rho) \otimes \mathbb{R} \cong$ $H_{i}\left(\widetilde{X}^{\infty} ; \mathbb{R}\right)$ is called the $i$-Alexander module of the $n$-knot $K$ (over $\mathbb{R}[\mathbb{Z}]$ ). Note that we can regard $H_{i}(X ; \rho) \otimes \mathbb{R} \cong H_{i}\left(\widetilde{X}^{\infty} ; \mathbb{R}\right) \cong\left(\mathbb{R}[\mathbb{Z}] / \lambda_{1}\right) \oplus \ldots \oplus\left(\mathbb{R}[\mathbb{Z}] / \lambda_{\mu_{i}}\right)$. We define the $i$-Alexander polynomial $\Lambda_{K, i}$ of the $n$-knot $K @$ to be $\lambda_{1} \cdot \ldots \cdot \lambda_{\mu_{i}}$. We must regard that $\Lambda_{K, i}(t)$ is same as $r \cdot t^{l} \cdot \Lambda_{K, i}(t)$, where $r \neq 0, r \in \mathbb{R}$ and $l \in \mathbb{Z}$. If the $i$-Alexander polynomial $\Lambda_{K, i}(t)$ can be represented by $a_{0}+a_{1} \cdot t^{1}+\ldots+a_{\nu_{i}} \cdot t^{\nu_{i}}\left(\nu_{i} \in \mathbb{N} \cup\{0\}, a_{0} \neq 0\right.$, $a_{\nu_{i}} \neq 0$.), then we say that the degree of the $i$-Alexander polynomial $\Lambda_{K, i}(t)$ is $\nu_{i}$. Note that the degree $\operatorname{deg} \Lambda_{K, i}(t)$ of the $i$-Alexander polynomial $\Lambda_{K, i}(t)$ coincides with the $i$ betti number of $\widetilde{X}^{\infty}$. (Recall that the $i$ betti number of $\widetilde{X}^{\infty}$ is the rank of the $\mathbb{R}$ vector space $H_{i}\left(\widetilde{X}^{\infty} ; \mathbb{R}\right)$.)

Note: There are many other invariants for $n$-knots and $n$-links. See e.g. [4] [8] [12] [14] [15] 16] 17] 18] 19] 20] 21].

By using results on homology with twisted coefficients and those on covering spaces, we can find many results on our Witten indexes. Some of them are the following.

(1) If one of our Witten indexes for an $n$-knot $K$ is nonzero, then one of the betti numbers of $\widetilde{X}^{\infty}$ is nonzero. Hence one of the Alexander polynomials of $K$ is nontrivial. Hence one of the betti numbers of the homology $H_{i}(X ; \rho)$ with the twisted coefficient defined by $\rho$ is nonzero.

(2) Let $K$ be a 1 -knot. Let $K$ be the trefoil knot. Then the $(0,1 ; 6)$-Witten index is not zero. Let $K^{(n+1)}$ be an $(n+1)$-knot which is the spun-knot of an $n$-knot 
$K^{(n)}(n \in \mathbb{N})$. Let $K^{(1)}$ be the trefoil knot. (See [22], 26] for spun-knot.) Then the $(0,1 ; 6)$-Witten index for $K^{(n)}(n \in \mathbb{N})$ is not zero.

Let $K$ be a simple $(4 k+1)$-knot $(k>0)$. (See e.g. [8] [12] [22] for simple knots.) Suppose that one of Seifert matrixes for $K$ is $\left(\begin{array}{ll}1 & 1 \\ 0 & 1\end{array}\right)$. Note that $K$ is not a trivial knot. Then the $(2 k, 2 k+1 ; 6)$-Witten index is not zero.

(3) Let $K$ be a 1-knot. Let $K$ be the figure eight knot. Then all of our Witten indexes are zero. Let $K^{(n+1)}$ be an $(n+1)$-knot which is the spun-knot of an $n$-knot $K^{(n)}(n \in \mathbb{N})$. Let $K^{(1)}$ be the figure eight knot. Then all of our Witten indexes are zero.

Let $K$ be a simple $(4 k+1)$-knot $(k>0)$. Suppose that one of Seifert matrixes for $K$ is $\left(\begin{array}{cc}1 & 1 \\ 0 & -1\end{array}\right)$. Note that $K$ is not a trivial knot. Then all of our Witten indexes are zero.

Note: In order to let the path integral make sense (in physics level), it might be better that we suppose $\pi_{1}\left(M^{\mu}\right)=1$ etc. See e.g. $\S 7$ of [1]. Note that there are countably infinitely many nontrivial $n$-knots $K$ with $\pi_{1}\left(M^{\mu}\right)=1$ for all $\mu$. For any simple $n$-knots $K$ and any $\mu$, it holds that $\pi_{1}\left(M^{\mu}\right)=1$.

Note: Our way gives an interpretation of the betti numbers of the homology with a twisted coefficient by using supersymmetric quantum theory not only when we discuss $n$-dimensional knot but also when we discuss other manifolds.

Note: In our model we consider the branched covering $M^{\mu}$ along an $n$-knot $K$ over $S^{n+2}$. $M^{\mu}$ are many kinds of $(n+2)$-manifolds. The manifold $M^{\mu}$ may give a candidate for a fiber if we regard our time-space as the fiber bundle $\mathbb{R}^{4} \times M$ with the base sapce $\mathbb{R}^{4}$ and with the fiber $M$. In this model we suppose that we can usually observe $\mathbb{R}^{4} \times M$ as $\mathbb{R}^{4}$ since $M$ is very small. Anyway we have the following if $M^{\mu}$ is a 6-dimensional or 7-dimensional manifold.

(4) Let $K$ be a 4-dimensional knot in $S^{6}$. Then all of our supersymmetries are broken if the all Alexander polynomials of $K$ are zero.

(5) Let $K$ be a 5 -dimensional knot in $S^{7}$. Then it holds: the $(4,3 ; q)$-Witten index for any $q$ is zero and there are countably infinitely many 5 -knots such that supersymmetry assciated with the $(4,3 ; q)$-Witten index are broken (resp. not broken). 


\section{REFERENCES}

[1] O. Alvarez: Lectures on quantum mechanics and the index theorem. Geometry and quantum field theory (Park City, UT, 1991), 271-322, IAS/Park City Math. Ser., 1, Amer. Math. Soc., Providence, RI, 1995.

[2] L. Alvarez-Gaumé: Supersymmetry and the Atiyah-Singer index theorem Communications in Mathematical Physics 90, 161-173, 1983.

[3] M. F. Atiyah and I. M. Singer: The index of elliptic operators. I. II. III. Ann. of Math. (2) 87 1968 484-604, IV. V. Ann. of Math. (2) 931971 119-149.

[4] T. D. Cochran and K. E. Orr: Not all links are concordant to boundary links Ann. of Math., $138,519-554,1993$.

[5] J. Davis and P. Kirk: Lecture notes in algebraic topology. Graduate Studies in Mathematics, 35. American Mathematical Society, Providence, RI, 2001.

[6] G. de Rham: Differentiable manifolds. Translated from the French by F. R. Smith. With an introduction by S. S. Chern. Grundlehren der Mathematischen Wissenschaften [Fundamental Principles of Mathematical Sciences], 266. Springer-Verlag, Berlin, 1984.

[7] A. Einstein: A paper whose content we call general theory of relativity, 1915

[8] M. Farber: Classification of simple knots. (Russian) Uspekhi Mat. Nauk 38, no. 5(233), 59-106, 1983.

[9] M. B. Green, J. H. Schwarz, and E. Witten: Suoerstring theory. VOL.1,2. Cambridge Monographs On Mathematical Physics. 1987.

[10] M. Kervaire: Les noeudes de dimensions supéreures, Bull.Soc.Math.France 93, 225-271, 1965.

[11] J. Levine: Knot cobordism in codimension two, Comment. Math. Helv., 44, 229-244, 1969.

[12] J. Levine: An algebraic classification of some knots of codimension two. Comment. Math. Helv. 45, 185-198, 1970.

[13] J. Levine: Polynomial invariants of knots of codimension two. Ann. of Math. 84, 537-554, 1966.

[14] J. Levine and K. Orr: A survey of applications of surgery to knot and link theory. Surveys on surgery theory: surveys presented in honor of C.T.C. Wall Vol. 1, 345-364, Ann. of Math. Stud., 145, Princeton Univ. Press, Princeton, NJ, 2000.

[15] E. Ogasa: Ribbon moves of 2-links preserve the $\mu$-invariants of 2-links University of Tokyo Preprint series, UTMS 97-35, 1997, math.GT/0004008.

[16] E. Ogasa: Intersectional pairs of $n$-knots, local moves of $n$-knots and invariants of $n$-knots Mathematical Research Letters, 1998, vol5, 577-582, UTMS 95-50

[17] E. Ogasa: The projections of $n$-knots which are not the projection of any unknotted knot Journal of knot theory and its ramifications, 10 (2001), no. 1, 121-132 UTMS 97-34, math.GT/0003088

[18] E. Ogasa: Singularities of projections of $n$-dimensional knots of Cambridge Philosophical Society 126, 1999, 511-519

Mathematical Proceedings

[19] E. Ogasa: The Alexander polynomials of $n$-knots and local moves UTMS 97-63, 1997

[20] E. Ogasa: $n$-dimensional links, their components, and their band-sums math.GT/0011163, UTMS00-65, 2000

[21] E. Ogasa: Ribbon-moves of 2-knots: The Farber-Levine pairing and the Atiyah-Pathodi-SingerCasson-Gordon-Ruberman $\eta$ invariant of 2-knots math.GT/0004007, UTMS 00-22, 2000

[22] D. Rolfsen: Knots and links Publish or Perish, Inc. 1976.

[23] M. S. Swanson: Path integrals and quantum processes. Academic Press, Inc., Boston, MA, 1992. 
[24] J. Wess and J. Bagger: Supersymmetry and supergravity Princeton Series in Physics 1992.

[25] E. Witten: Supersymmetry and Morse theory Journal of Differential Geometry 117, 353-386, 1982.

[26] E. Zeeman: Twisting spun knots, Trans. Amer. Math. Soc., 115, 471-495, 1965.

Acknowledgment. The author would like to thank Prof. Tohru Eguchi, Prof. Akio Sugamoto, Prof. Tomoyoshi Yoshida, Prof. Yutaka Matsuo, Dr. Kentaro. Hori, Dr. Yukiko Konishi for their encouragement and for their interest.

High Energy Physics Theory Group, Department of Physics, Ochanomizu University, BunKYO-KU, TOKYO 112-8610, JAPAN,

E-mail address: ogasa@hep-th.phys.u-tokyo.ac.jp 